\title{
PARCERIA ENTRE GRANDES EMPRESAS E STARTUPS QUE PARTICIPAM DE ACELERADORAS CORPORATIVAS: BENEFÍCIOS E DESVANTAGENS PARA AS STARTUPS
}

\author{
PARTNERSHIP BETWEEN LARGE COMPANIES AND STARTUPS THAT PARTICIPATE IN \\ CORPORATE ACCELERATORS: BENEFITS AND DISADVANTAGES FOR STARTUPS
}

Recebido em 29.10.2020 Aprovado em 06.11.2020
Avaliado pelo sistema double blind review
DOI: https://doi.org/10.32888/cge.v8i3.46985

Mayara Kasai Yoshiyassu

mayara.yoshiyassu@usp.br

Universidade de São Paulo, Faculdade de Economia, Administração e Contabilidade de Ribeirão Preto, Ribeirão

Preto, SP, Brasil

https://orcid.org/0000-0003-3279-3335

\section{Alexandre Rodrigues Cajuela}

alexandrecajuela@usp.br

Universidade de São Paulo, Faculdade de Economia, Administração e Contabilidade de Ribeirão Preto, Ribeirão

Preto, SP, Brasil

https://orcid.org/0000-0002-9836-8676

\section{Bruna Toffoli Affonso}

btoffoliaffonso@gmail.com

Universidade de São Paulo, Faculdade de Economia, Administração e Contabilidade de Ribeirão Preto, Ribeirão

Preto, SP, Brasil

https://orcid.org/0000-0003-0728-3755

\section{Simone Vasconcelos Ribeiro Galina}

svgalina@usp.br

Universidade de São Paulo, Faculdade de Economia, Administração e Contabilidade de Ribeirão Preto, Ribeirão

Preto, SP, Brasil

https://orcid.org/0000-0001-7150-2217

\section{Resumo}

O objetivo deste trabalho é avaliar a percepção das startups que participam de aceleradoras corporativas quanto aos benefícios de capacidade de absorção decorrentes do relacionamento com uma grande empresa, e também descrever as desvantagens dessa parceria. A pesquisa é quantitativa e descritiva, com método de levantamento do tipo survey. Os resultados mostram que as aceleradoras corporativas classificadas como programas estruturados de inovação geram capacidade de absorção às startups em maior escala. A principal desvantagem foi a complexidade de gestão. Conclui-se que o maior apoio oferecido às startups em programas estruturados resulta em maiores benefícios.

Palavras-chave: Relacionamento interorganizacional. Aceleradoras corporativas. Benefícios. Capacidade de absorção. Desvantagens. Ecossistemas de inovação.

\begin{abstract}
The objective of this study is to assess the perception of startups that participate in corporate accelerators as to the absorptive capacity benefits arising from the relationship with a large company, and also to describe the disadvantages of this partnership. This research is quantitative and descriptive, with a survey method. The results show that corporate accelerators classified as structured innovation programs generate absorptive capacity for startups in a higher scale. The main disadvantage was management complexity. The conclusion is that the greater support offered to startups in structured programs result in greater benefits.
\end{abstract}

Keywords: Interorganizational relationship. Corporate accelerators. Benefits. Absorptive capacity. Disadvantages. Innovation ecosystems. 


\section{Introdução}

No contexto dos ecossistemas de inovação, as grandes empresas têm aumentado seu envolvimento com startups nos últimos anos (Groote \& Backmann, 2020) com o objetivo de complementar recursos internos, ampliar o escopo e a velocidade da sua capacidade de inovação e geração de valor, atender as expectativas dos consumidores e sustentar uma vantagem competitiva (Weiblen \& Chesbrough, 2015). Isso porque a maioria das corporações apresentam dificuldades para inovar isoladamente e buscam complementar suas competências internas (Jang, Lee, \& Yoon, 2017). Assim, as startups possuem recursos que geralmente interessam às grandes empresas e podem contribuir para a inovação e a competitividade dessas organizações (Brink, 2017). Da mesma forma, as vantagens dessa aproximação também podem ser observadas pelas startups, que se beneficiam de alianças com grandes empresas por meio de melhorias nos seus sistemas de gestão, nas suas condições financeiras e, consequentemente, no desenvolvimento de vantagem competitiva (Wasiuzzaman, 2019).

Uma forma usual e crescente de aproximar startups e grandes empresas é por meio dos programas de aceleração liderados por corporações consolidadas, os quais, desde o início de 2010, aumentaram em todo o mundo (Moschner et al., 2019; Shankar \& Shepherd, 2019; Urbaniec \& Żur, 2020). Estudos empíricos mostram que as aceleradoras corporativas possibilitam, dentre outros, a prestação de suporte para aumentar o acesso e o crescimento das startups no mercado (Crișan et al., 2019), bem como o desenvolvimento de capacidades em startups, destacando a capacidade de absorção de conhecimento (Cajuela \& Galina, 2020).

Segundo Kohler (2016), aceleradoras corporativas são programas apoiados por grandes empresas que possuem duração limitada e dão suporte a conjuntos de startups durante o seu processo de desenvolvimento. As aceleradoras corporativas geralmente procuram explorar o conhecimento e a inovação das startups à medida que as auxiliam a resolver problemas internos ou criam oportunidades de crescimento (Moschner et al., 2019), por meio de mentorias, educação e recursos específicos (Kohler, 2016).

No entanto, a literatura apresenta predominantemente estudos sobre esses programas do ponto de vista das grandes corporações, e não das startups (Dooley, Kenny, \& Cronin, 2015; Evangelista \& Mac, 2016). Alguns questionamentos são evidentes, como a falta de clareza sobre a eficácia desses programas de aceleração, conforme vão se proliferando pelo mundo (Cohen \& Hochberg, 2014). Portanto, nota-se que a pesquisa sobre o impacto das aceleradoras corporativas nas startups tem sido anêmica (Cohen \& Hochberg, 2014), indicando que esses tipos de relacionamento entre grandes empresas e startups ainda precisam ser investigados para encontrar novos conceitos e avaliar seus resultados.

Ao analisar os diferentes serviços que as aceleradoras oferecem junto às startups, Crișan et al. (2019) classificaram as intervenções como restrita, típica e ampliada. As startups que participam da intervenção restrita têm acesso a poucas opções de serviços, como suporte à comunidade virtual, workshops e palestras, mesas-redondas de estratégia, além de espaço físico para suas operações. Já nas intervenções típica e ampliada, as startups recebem maior apoio em gestão, orientação de mentores, treinamentos frequentes e, considerando o contexto das aceleradoras corporativas, acesso a recursos e parceiros das grandes empresas responsáveis pelos programas. As aceleradoras corporativas brasileiras oferecem intervenções semelhantes às classificadas por Crișan et al. (2019), podendo ser agrupadas em espaços de inovação (restrita) e programas estruturados de inovação (típica e ampliada). Diante disso, é possível que haja diferença na percepção das startups em cada um desses tipos de aceleradoras corporativas quanto aos benefícios gerados pela relação com a grande empresa.

Portanto, o objetivo deste estudo é avaliar a parceria entre grandes empresas e startups que participam de aceleradoras corporativas, buscando descrever se os tipos de apoio fornecidos pela grande empresa nos espaços de inovação e nos programas estruturados de inovação proporcionam às startups diferentes benefícios. $\mathrm{O}$ estudo procura entender se, nos programas estruturados de inovação, as relações com as grandes empresas possibilitam às startups adquirir novos conhecimentos, estabelecer rotinas eficazes de comunicação com a grande empresa, aplicar os conhecimentos adquiridos no trabalho prático e utilizá-los para o desenvolvimento de novos produtos e serviços ou na melhoria do desenvolvimento da própria startup. 
Apesar dos benefícios esperados para as startups ao participarem de aceleradoras corporativas, é possível que haja dificuldades em função das diferenças existentes em relação às corporações. Algumas potenciais desvantagens de um relacionamento interorganizacional foram definidas por Barringer e Harrison (2000) e podem ser observadas também entre grandes empresas e startups. Portanto, este trabalho também visa descrever as desvantagens da parceria com uma grande empresa percebidas pelas startups que participam de aceleradoras corporativas brasileiras.

\section{Referencial teórico}

\section{Relacionamentos interorganizacionais entre grandes empresas e startups}

A formação de relacionamentos interorganizacionais envolve, geralmente, uma mistura de motivos, intenções e objetivos relacionados a seis paradigmas teóricos: economia de custos de transação, dependência de recursos, escolha estratégica, teoria das partes interessadas, teoria da aprendizagem e teoria institucional (Barringer \& Harrison, 2000), que são utilizados para alcançar os objetivos organizacionais (Becker \& Dietz, 2004). Além desses paradigmas, as organizações estão caminhando para um modelo de inovação mais colaborativo e em rede, onde empresas de portes ou maturidades distintas estão propensas a se relacionarem como parte de sua estratégia de inovação (Dooley, Kenny, \& Cronin, 2015).

A conexão entre grandes empresas e startups é motivada, por parte das grandes corporações, pelos benefícios trazidos, como mentalidade empreendedora, soluções inovadoras (Sommer, Heidenreich, \& Handrich, 2017), conhecimentos específicos, flexibilidade, criatividade e maior rapidez para responder a mudanças (Weiblen \& Chesbrough, 2015; Simon \& Leker, 2016). As startups podem, em contrapartida, ter acesso a recursos e clientes das grandes empresas, aumentando as suas chances de crescimento (Usman \& Vanhaverbeke, 2017), ter contato direto com os modelos de gestão das grandes empresas e, com isso, melhorar sua condição financeira e vantagem competitiva (Wasiuzzaman, 2019), além do acesso a tecnologias líderes que as grandes empresas podem proporcionar (Stuart, 2000). Uma forma de promover a conexão entre essas organizações é por meio das aceleradoras corporativas, que são fomentadas ou apoiadas por empresas já estabelecidas no mercado.

\section{Aceleradoras Corporativas}

Os programas de aceleração de empresas gerenciados ou patrocinados diretamente por uma ou várias empresas estabelecidas, as chamadas aceleradoras corporativas, estão se tornando parte integrante dos ecossistemas de startups e um importante veículo de engajamento de relacionamentos interorganizacionais (Moschner et al., 2019). Nesse contexto, as aceleradoras, cuja atividade pode ser considerada um fenômeno extremamente recente no mundo (Pauwels et al., 2016), desempenham um papel importante no estímulo ao empreendedorismo. O estudo de Abreu e Campos (2016) localizou 45 aceleradoras de empresas presentes no ecossistema empreendedor brasileiro, envolvendo empresas líderes, que se utilizam dos relacionamentos com múltiplos parceiros para criar inovações mais amplas e influentes (Davis, 2016).

Considerando os serviços oferecidos pelas aceleradoras às startups, Crișan et al. (2019) identificaram três tipos de intervenções: restrita, típica e ampliada. Elas se diferenciam, basicamente, por proporcionarem maior ou menor envolvimento entre a grande corporação e as startups, sendo que as do tipo restrita oferecem poucas opções de serviços além de espaço físico; as do tipo típica e ampliada já estabelecem apoio em gestão, orientação, treinamentos e acesso a recursos e parceiros das grandes corporações. Considerando esse estudo, pode-se classificar as aceleradoras corporativas brasileiras em basicamente dois tipos, que são chamadas aqui de "espaços de inovação" e "programas estruturados de inovação". Considerados menos comuns, os espaços de inovação assemelham-se ao tipo de intervenção restrita (Crișan et al., 2019) e fornecem um pacote limitado de serviços, como suporte à comunidade virtual, workshops e palestras, espaços fixos de trabalho, salas de reunião, dentre outros. Já os programas estruturados de inovação fornecem, além dos serviços dos espaços de inovação, oportunidades de negócios com a empresa estabelecida, sua rede de parceiros e clientes, networking com executivos e investidores, 
assistência em questões técnicas, jurídicas e financeiras, mentorias e treinamentos, e outros serviços específicos. Percebe-se que os programas estruturados de inovação se equiparam aos tipos de intervenção típica e ampliada identificadas por Crișan et al. (2019), considerados os mais populares e que oferecem uma ampla gama de serviços para possibilitar estreitamento da relação entre a grande empresa e a startup, sendo que o foco principal é o processo de mentoria (Abreu \& Campos, 2016) e o desenvolvimento de rotinas organizacionais específicas e processos que operacionalizam e potencializam a aprendizagem organizacional (Cajuela \& Galina, 2020).

O estudo de Urbaniec e Żur (2020) evidenciou que as aceleradoras corporativas possuem potencial para trazer benefícios diretos e indiretos tanto para as grandes empresas quanto para as startups. O principal benefício para ambas as empresas envolve, a curto prazo, novos conhecimentos sobre o mercado, relacionados às expectativas dos clientes, tendências do setor, novas tecnologias e concorrência. Os autores supracitados destacam que o benefício estratégico mais importante e de longo prazo é o aprendizado organizacional, decorrente desse novo acúmulo de conhecimento.

\section{Benefícios e desvantagens para as startups}

Como mencionado, um dos benefícios mais importantes do relacionamento interorganizacional é o desenvolvimento da capacidade de absorver conhecimento externo e combiná-lo com o conhecimento interno já existente (Zahra \& George, 2002; Liu \& Yang, 2019), ou seja, a aprendizagem organizacional. Essa capacidade, chamada de capacidade de absorção, explica o processo de aprendizagem organizacional (Cohen \& Levinthal, 1990) e está relacionada à aquisição, assimilação, transformação e exploração de recursos e conhecimentos, sendo as duas primeiras referentes à capacidade de absorção potencial, e as duas últimas, à capacidade de absorção realizada (Zahra \& George, 2002). Em especial, a inter-relação de startups com grandes empresas em aceleradoras corporativas potencializa o desenvolvimento da capacidade de absorção potencial nas startups, pois facilita o acesso e a assimilação de conhecimento (Cajuela \& Galina, 2020).

O estudo de Pavlou e El Sawy (2006) utilizou uma escala com dez itens para medir a capacidade de absorção na área de desenvolvimento de novos produtos, avaliando a aquisição, assimilação, transformação e exploração eficazes do conhecimento. Da perspectiva dessa escala, os benefícios para as startups em relação à aprendizagem organizacional avaliados neste estudo estão relacionados à aquisição de novos conhecimentos, estabelecimento de rotinas eficazes de comunicação com a grande empresa, aplicação dos novos conhecimentos adquiridos ao trabalho prático da startup e utilização das informações e conhecimentos obtidos da grande empresa no desenvolvimento de novos produtos e serviços ou na melhoria do desempenho da startup.

Tendo em vista que existe na literatura uma lacuna em relação aos benefícios proporcionados às startups por diferentes serviços em aceleradoras (Choi \& Kim, 2018), mais especificamente quanto aos efeitos no processo de aprendizagem organizacional (Cohen, Fehder, Hochberg, \& Murray, 2019; Crișan et al., 2019), um dos objetivos desta pesquisa é avaliar se os benefícios para as startups diferem de acordo com o suporte e serviço que recebem da aceleradora corporativa. Diante disso, foram definidas as seguintes hipóteses:

H1: em comparação com as startups que estão em espaços de inovação, aquelas inseridas em programas estruturados de inovação usam mais a parceria para aquisição de novos conhecimentos;

H2: em comparação com as startups que estão em espaços de inovação, aquelas inseridas em programas estruturados de inovação usam mais a parceria para estabelecer rotinas eficazes de comunicação com a grande empresa;

H3: em comparação com as startups que estão em espaços de inovação, aquelas inseridas em programas estruturados de inovação usam mais a parceria para aplicação dos novos conhecimentos adquiridos da grande empresa ao trabalho prático da startup;

H4: em comparação com as startups que estão em espaços de inovação, aquelas inseridas em programas estruturados de inovação aproveitam mais a parceria para utilizar as informações e conhecimentos obtidos da grande empresa no desenvolvimento de novos produtos e serviços ou na melhoria do desempenho. 
Entretanto, é relevante considerar que há desafios e dificuldades nessa relação entre grandes corporações e startups, especialmente por causa dos conflitos inerentes às diferenças e peculiaridades de cada uma dessas organizações como tamanho, cultura, enrijecimento estrutural, dentre outros. Não obstante, Barringer e Harrison (2000) concentraram seus estudos nas desvantagens potenciais das relações interorganizacionais, destacando a perda de informações relacionadas a propriedade intelectual; a complexidade de gestão; os riscos financeiros e organizacionais; o risco de tornar-se dependente de um parceiro; a perda parcial da autonomia de decisão; o conflito entre a cultura organizacional de cada parceiro; a perda de flexibilidade organizacional; e as implicações antitruste. Deste modo, este estudo também buscou descrever as desvantagens percebidas pelas startups inseridas em aceleradoras corporativas, seja nos espaços de inovação ou nos programas estruturados.

\section{Procedimentos metodológicos}

A pesquisa seguiu uma abordagem quantitativa para descrever a opinião das startups inseridas em programas estruturados de inovação ou espaços de inovação, ambos promovidos por grandes empresas. Foi utilizada a pesquisa de levantamento como método de pesquisa, e a coleta dos dados foi realizada por meio de questionários aplicados via e-mail e LinkedIn, utilizando a escala do tipo Likert para medir o grau de concordância dos participantes em relação às afirmações. Para a elaboração do questionário, a escala de Pavlou e El Sawy (2006) serviu como base para as perguntas cujo objetivo foi analisar os benefícios para as startups em relação à aprendizagem organizacional e a escala de Barringer e Harrison (2000) para as desvantagens na relação com as grandes empresas. Os questionários foram aplicados entre outubro e dezembro de 2017, e maio e junho de 2018.

A busca das aceleradoras corporativas foi feita primeiramente em portais on-line de conteúdo sobre empreendedorismo, os quais noticiam programas de inovação desenvolvidos por diversas empresas. Identificouse que os termos mais utilizados são "programa de aceleração de startups", "programa de apoio a startups", "espaço de inovação e empreendedorismo", "aceleradora corporativa”; desta forma, o segundo passo foi a inserção dessas palavras-chave no buscador Google.

A seleção das aceleradoras corporativas considerou os fatores: possuir website oficial, oferecer acesso a informações sobre seus serviços, estar em funcionamento e disponibilizar no site a lista de startups que passaram pelo programa. A partir desse filtro, foram obtidas dez aceleradoras corporativas cujos serviços foram analisados, conforme o Quadro 1, resultando na identificação de oito programas estruturados de inovação (Google Launchpad, Wayra, Oxigênio, Braskem Labs, TegUp, Liga Autotech, Liga Emerging Technologies e InovaBra) e dois espaços de inovação (Cubo e Google Campus São Paulo).

Quadro 1 - Descrição e classificação das aceleradoras corporativas brasileiras selecionadas

\begin{tabular}{|c|c|c|}
\hline $\begin{array}{l}\text { Aceleradora } \\
\text { corporativa }\end{array}$ & Serviços oferecidos & Classificação \\
\hline $\begin{array}{l}\text { InovaBra } \\
\text { Startups }\end{array}$ & $\begin{array}{l}\text { Realização de prova de conceito com o Bradesco, validação do negócio na } \\
\text { prática, análise das soluções por especialistas do banco, preparação para } \\
\text { captar investimentos, espaço para instalação, mentorias e possibilidades de } \\
\text { negócio com os clientes e parceiros do Bradesco. }\end{array}$ & $\begin{array}{l}\text { Programa estruturado de } \\
\text { inovação }\end{array}$ \\
\hline Braskem Labs & $\begin{array}{l}\text { Há dois programas de aceleração: Ignition e Scale. O Scale é para startups com } \\
\text { soluções já validadas, oferecendo capacitação, possibilidade de parceria com } \\
\text { a Braskem ou clientes, acesso a equipes de inovação internas, rede de } \\
\text { contatos estratégicos e apresentação para investidores no Demo Day. O } \\
\text { Ignition é para startups em fase de validação ou crescimento, oferecendo } \\
\text { acompanhamento de marketing e vendas, acesso a investidores e os } \\
\text { mesmos benefícios do Scale, com exceção do Demo Day. }\end{array}$ & $\begin{array}{l}\text { Programa estruturado de } \\
\text { inovação }\end{array}$ \\
\hline
\end{tabular}




\begin{tabular}{|c|c|c|}
\hline $\begin{array}{l}\text { Aceleradora } \\
\text { corporativa }\end{array}$ & Serviços oferecidos & Classificação \\
\hline Cubo & $\begin{array}{l}\text { Para empresa residente, oferece recursos disponíveis na plataforma digital do } \\
\text { Cubo, visibilidade de marca e prioridade para realização de eventos, } \\
\text { participação em eventos do Cubo, salas de reunião e posiçôes de trabalho. A } \\
\text { categoria membro oferece exposição aos residentes e parceiros do Cubo; } \\
\text { espaços de trabalho e salas de reunião; recursos disponíveis na plataforma } \\
\text { digital e possibilidade de se tornar residente. }\end{array}$ & Espaço de inovação \\
\hline $\begin{array}{l}\text { Google } \\
\text { Campus São } \\
\text { Paulo }\end{array}$ & $\begin{array}{l}\text { No Programa de Residência, oferece às startups } 6 \text { meses de espaço de } \\
\text { trabalho permanente, acesso prioritário à rede de experts do Google, } \\
\text { treinamentos e mentorias inteiramente customizados para cada uma das } \\
\text { startups participantes, oportunidades para participar de programas de imersão } \\
\text { globais, além de eventos e conteúdos exclusivos. }\end{array}$ & Espaço de inovação \\
\hline $\begin{array}{l}\text { Google } \\
\text { Launchpad } \\
\text { Accelerator }\end{array}$ & $\begin{array}{l}\text { As startups recebem suporte livre de equity, duas semanas de treinamento } \\
\text { com todas as despesas pagas no Google HQ no Vale do Silício, orientação } \\
\text { personalizada de especialistas do Google e do Vale do Silício, créditos dos } \\
\text { produtos do Google, mídia global e oportunidades de destaque em } \\
\text { marketing, suporte contínuo por seis meses após a conclusão do } \\
\text { programa, acesso a uma comunidade global de fundadores e mentores do } \\
\text { estágio de crescimento. }\end{array}$ & $\begin{array}{l}\text { Programa estruturado de } \\
\text { inovação }\end{array}$ \\
\hline Liga Autotech & $\begin{array}{l}\text { Envolve mais de uma corporação e oferece mentorias gerais e específicas, } \\
\text { acompanhamento customizado e diversos outros benefícios. Não há } \\
\text { investimento em dinheiro nem compra de equity. }\end{array}$ & $\begin{array}{l}\text { Programa estruturado de } \\
\text { inovação }\end{array}$ \\
\hline Oxigênio & $\begin{array}{l}\text { Oferece investimento direto de US } \$ 50.000,00 \text { por } 10 \% \text { de participação, } \\
\text { aceleração por três meses no Centro de Inovação da Oxigênio em São Paulo } \\
\text { e três meses na sede da Plug and Play, no Vale do Silício, oportunidade de } \\
\text { fazer negócios com empresas do grupo Porto Seguro, mentorias com } \\
\text { grandes nomes do mercado, acesso a serviços e ferramentas com } \\
\text { condições especiais. }\end{array}$ & $\begin{array}{l}\text { Programa estruturado de } \\
\text { inovação }\end{array}$ \\
\hline $\begin{array}{l}\text { TegUp } \\
\text { Ventures }\end{array}$ & $\begin{array}{l}\text { O programa oferece acesso ao networking da Tegma, coaching e mentoria, } \\
\text { espaço de coworking, laboratório real para testes, apoio à gestão e } \\
\text { investimento. }\end{array}$ & $\begin{array}{l}\text { Programa estruturado de } \\
\text { inovação }\end{array}$ \\
\hline Wayra Brasil & $\begin{array}{l}\text { O programa oferece mentoria, apoio para receber financiamento, espaço de } \\
\text { trabalho, conexão com a rede global, acesso a empreendedores e } \\
\text { investidores e a possibilidade de acesso a mercados. }\end{array}$ & $\begin{array}{l}\text { Programa estruturado de } \\
\text { inovação }\end{array}$ \\
\hline $\begin{array}{l}\text { Liga Emerging } \\
\text { Technologies }\end{array}$ & $\begin{array}{l}\text { Envolve mais de uma corporação e oferece mentorias gerais e específicas, } \\
\text { acompanhamento customizado e diversos outros benefícios. Não há } \\
\text { investimento em dinheiro nem compra de equity. }\end{array}$ & $\begin{array}{l}\text { Programa estruturado de } \\
\text { inovação }\end{array}$ \\
\hline
\end{tabular}

Em seguida, foi realizado o mapeamento das startups de tais programas e espaços. Para os casos em que não havia disponível uma forma de contato, buscou-se no Google por "fundador da startup (nome da startup)", de modo a aparecer o perfil do LinkedIn ou alguma notícia que indicasse informações para ajudar a encontrar seu contato. A somatória de todas as startups dos programas selecionados foi 283 , mas foram excluídas as repetições daquelas que haviam participado de mais de um programa, resultando em 264 startups. Nesse caso, elas foram enquadradas no programa e/ou espaço que havia sido mais recente. Das 264, 21 não possuíam endereço eletrônico ou não foram encontradas, restando 243 startups, que foram convidadas a responder o questionário survey online, criado na plataforma LimeSurvey.

As variáveis referentes aos benefícios da parceria com a grande empresa, relacionadas à capacidade de absorção, foram adaptadas de Pavlou e El Sawy (2006) e estão descritas no Quadro 2. Elas têm como objetivo identificar a 
capacidade de absorção das startups, sendo que cada variável está relacionada respectivamente à aquisição, assimilação, transformação e exploração de recursos e conhecimentos.

Quadro 2 - Descrição das variáveis de benefícios relacionadas à capacidade de absorção

\begin{tabular}{|l|l|}
\hline Variável & Descrição \\
\hline Adquirir-conhecimento & Aquisição de novos conhecimentos para a startup. \\
\hline Rotina-comunicação & Realização de reuniões periódicas com a grande empresa. \\
\hline Aplicar-conhecimento & $\begin{array}{l}\text { Aplicação dos novos conhecimentos adquiridos da grande empresa ao trabalho prático da } \\
\text { startup. }\end{array}$ \\
\hline Utilizar-conhecimento & $\begin{array}{l}\text { Utilização das informações e conhecimentos obtidos da grande empresa no desenvolvimento } \\
\text { de novos produtos e serviços ou na melhoria do desempenho da startup. }\end{array}$ \\
\hline
\end{tabular}

Fonte: Pavlou e El Sawy (2006).

Já as desvantagens potenciais de se manter um relacionamento com uma grande empresa, descritas no Quadro 3, foram definidas por Barringer e Harrison (2000). Elas abordam os riscos potenciais na relação de parceria e permitirão trazer informações da visão das startups sobre o assunto.

Quadro 3 - Descrição das variáveis de desvantagens

\begin{tabular}{|c|c|}
\hline Variável & Descrição \\
\hline $\begin{array}{l}\text { Perda-PI (Perda de } \\
\text { propriedade intelectual) }\end{array}$ & $\begin{array}{l}\text { Informações relacionadas à propriedade intelectual podem ser perdidas para um parceiro que } \\
\text { já é um concorrente ou pode se tornar um. }\end{array}$ \\
\hline $\begin{array}{l}\text { Complex-gestão } \\
\text { (Complexidade de gestão) }\end{array}$ & $\begin{array}{l}\text { Como as relações interorganizacionais exigem o esforço combinado de duas ou mais } \\
\text { empresas, elas frequentemente são difíceis de gerenciar, incorrendo em frustrações e atrasos } \\
\text { onerosos. }\end{array}$ \\
\hline $\begin{array}{lr}\text { Riscos-fin-org } & \text { Riscos } \\
\text { financeiros } & \mathrm{e} \\
\text { organizacionais }) & \end{array}$ & $\begin{array}{l}\text { Os relacionamentos interorganizacionais apresentam uma alta taxa de fracasso. Além disso, a } \\
\text { participação em relacionamentos interorganizacionais leva a possíveis comportamentos } \\
\text { oportunistas por parte do parceiro. }\end{array}$ \\
\hline $\begin{array}{l}\text { Riscos-depend (Riscos de } \\
\text { tornar-se dependente de } \\
\text { um parceiro) }\end{array}$ & $\begin{array}{l}\text { Um desequilíbrio de poder surge se um parceiro se torna excessivamente dependente do } \\
\text { outro. Esta situação aumenta o potencial de oportunismo na parte do parceiro mais forte. } \\
\text { Um desequilíbrio de energia também aumenta chances de que a aliança leve a uma aquisição. }\end{array}$ \\
\hline $\begin{array}{l}\text { Perda-autonomia (Perda } \\
\text { parcial da autonomia de } \\
\text { decisão) }\end{array}$ & $\begin{array}{l}\text { O planejamento conjunto e a tomada de decisões podem resultar em perda de autonomia de } \\
\text { decisão. }\end{array}$ \\
\hline $\begin{array}{l}\text { Conflito-cultura (Cultura } \\
\text { organizacional do parceiro } \\
\text { conflitante) }\end{array}$ & $\begin{array}{l}\text { A cultura corporativa do parceiro pode conflitar com a da startup, fazendo com que a } \\
\text { implementação e gestão da aliança seja difícil. }\end{array}$ \\
\hline $\begin{array}{l}\text { Perda-flexibilidade (Perda } \\
\text { de flexibilidade } \\
\text { organizacional) }\end{array}$ & $\begin{array}{l}\text { Estabelecer uma parceria com uma empresa pode impedir a parceria com outra. Além disso, } \\
\text { as rotinas organizacionais criadas por uma aliança podem dificultar que uma empresa aja de } \\
\text { forma independente. }\end{array}$ \\
\hline $\begin{array}{l}\text { Implica-antitruste } \\
\text { (Implicações antitruste) }\end{array}$ & $\begin{array}{l}\text { Os benefícios de um relacionamento interorganizacional podem ser severamente afetados se } \\
\text { forem contestados por razões antitruste. Além disso, alguns países têm leis antitruste rígidas } \\
\text { que proíbem certas relações comerciais entre empresas. }\end{array}$ \\
\hline
\end{tabular}

Fonte: Barringer e Harrison (2000, p. 386). 
O número de startups respondentes foi 60, diante da amostra de 243. Contudo, 3 responderam que não possuíam parceria com uma grande empresa, restando 57 respostas válidas, o que representa 23,45\% do total. As startups integrantes dos espaços de inovação - Cubo e Google Campus - foram denominadas de Grupo 1, enquanto o Grupo 2 foi composto por startups dos programas estruturados de inovação (Google Launchpad, Wayra, Oxigênio, Braskem Labs, TegUp, Liga Autotech, Liga Emerging Technologies e InovaBra). Do total de 57 respondentes, 22 startups foram classificadas no Grupo 1 e 35 no Grupo 2.

Visto que as escalas utilizadas eram tipo Likert, a análise adequada foi a de frequência, mediana e/ou moda ao invés da média (Boone \& Boone, 2012). Para a avaliação das hipóteses, foram gerados no software IBM SPSS Statistics 20 os gráficos boxplot, os quais permitiram verificar a distribuição de dados, a assimetria da distribuição e as medianas. Assim, para cada uma das variáveis de capacidade de absorção foram comparadas as respostas do Grupo 1 com o Grupo 2. Já em relação às variáveis de desvantagens da parceria com a grande empresa, foi realizada uma análise descritiva por meio do ranqueamento das medianas e modas.

\section{Apresentação e análise dos resultados}

Com intuito de atender ao objetivo e avaliar as hipóteses deste estudo, foi feita a comparação dos dois tipos de aceleradoras (espaços e programas estruturados). Os benefícios relacionados à capacidade de absorção foram percebidos de formas diferentes pelas startups que compõem essas distintas aceleradoras (Gráfico 1).

Gráfico 1 - Boxplot dos benefícios referentes à capacidade de absorção, comparando o Grupo 1 (Espaços de inovação) com o Grupo 2 (Programas estruturados de inovação)

A

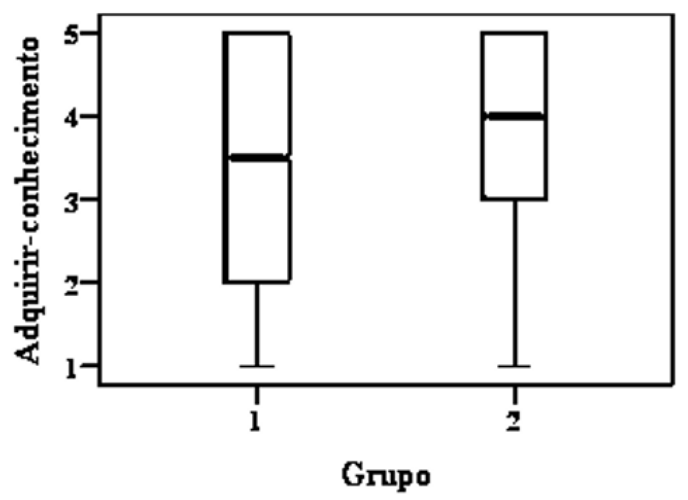

C

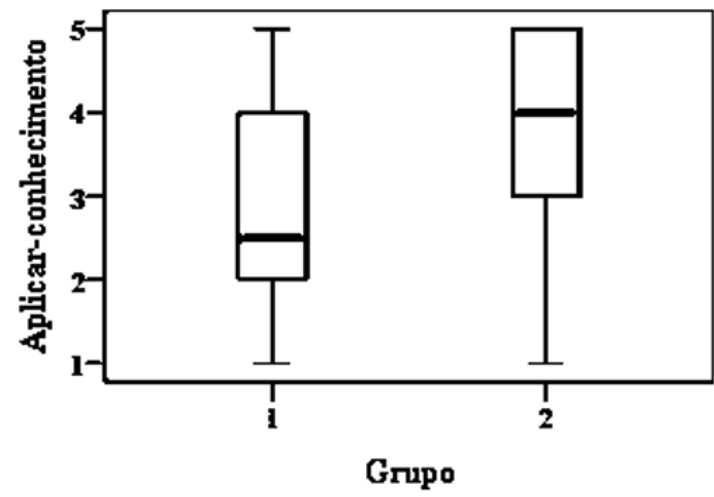

B

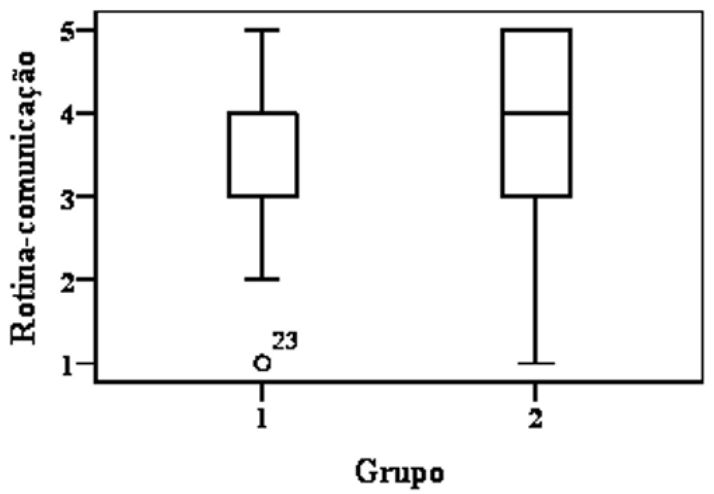

D

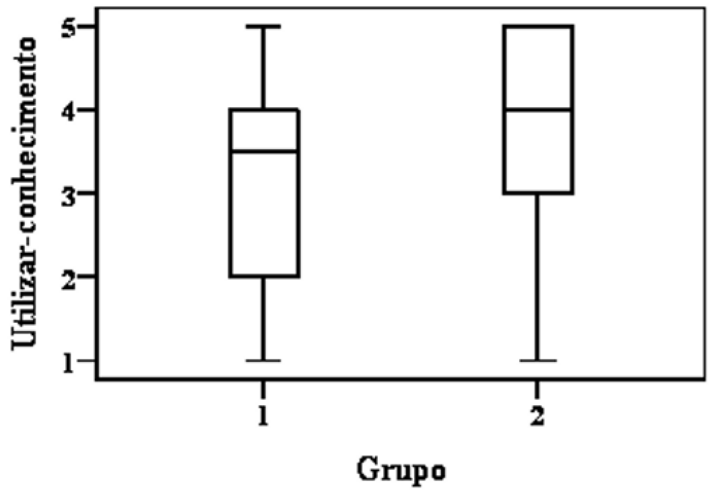

No Gráfico 1-A, verifica-se que o Grupo 1, formado pelas startups que fazem parte dos espaços de inovação, apresenta maior variabilidade de respostas, distribuídas entre 2 (raramente) e 5 (sempre), sendo a mediana de 3,5, 
o que significa que a aquisição de novos conhecimentos ocorre às vezes ou frequentemente. Quanto ao Grupo 2, composto por startups integrantes de programas estruturados de inovação, a mediana é igual a 4, indicando que a aquisição de novos conhecimentos ocorre frequentemente. Isso mostra que as startups do Grupo 2 utilizam a parceria com a grande empresa para aquisição de novos conhecimentos de forma mais frequente, em comparação com o Grupo 1, corroborando com o estudo de Cajuela e Galina (2020), uma vez que capacitações e redes de mentores disponibilizadas pelos programas estruturados possibilitam acesso a novas ideias, que são posteriormente avaliadas e aceitas ou não pelas startups. Analisando tais dados, pode-se dizer que, embora haja uma tendência maior das startups em programas estruturados a usar a parceria com uma grande empresa para aquisição de conhecimento, ela não é tão acentuada em comparação ao grupo 1, o que confirma parcialmente a hipótese 1.

Em seguida, no Gráfico 1-B, nota-se que ambos os grupos apresentam a mediana igual a 4, o que indica que a realização de reuniões periódicas com a grande empresa ocorre frequentemente, incentivando a discussão de novas ideias e conhecimentos relevantes, permitindo o compartilhamento de informações (Pavlou \& El Sawy, 2006). Essa comunicação efetiva facilita a assimilação do conhecimento, pois permite às startups processar e internalizar o conhecimento gerado externamente (Zahra \& George, 2002). Entretanto, o Grupo 1 apresenta respostas que variam entre 3 (às vezes) e 4 (frequentemente), enquanto as respostas do Grupo 2 variam entre 3 (às vezes) e 5 (sempre). Apesar da mediana dos dois grupos ser a mesma, o Grupo 2 apresenta uma maior frequência de respostas 5 , indicando que há uma tendência maior das startups que participam de programas estruturados de inovação a estabelecer rotinas eficazes de comunicação com a grande empresa. Portanto, a hipótese 2 pode ser considerada validada.

Ao analisar o Gráfico 1-C, verifica-se que no Grupo 1 há uma variabilidade de respostas entre 2 (raramente) e 4 (frequentemente), e a mediana 2,5 indica que a parceria com a grande empresa envolve a aplicação de conhecimento raramente ou às vezes. Por outro lado, as respostas do Grupo 2 variam entre 3 (às vezes) e 5 (sempre) sendo a mediana 4, indicando que a aplicação dos conhecimentos ocorre frequentemente. Sendo assim, há uma tendência maior das startups dos programas de inovação estruturados a aplicar os novos conhecimentos adquiridos da grande empresa ao trabalho prático da startup, o que valida a hipótese 3. Isso possivelmente se dá porque a relação formal e estruturada com a grande corporação permite à startup acesso a conhecimento de mercado que ela não teria como obter sozinha, conforme observado por Urbaniec e Żur (2020). A aplicação do conhecimento denota das startups sua capacidade de combinar conhecimentos existentes com o conhecimento recém adquirido e assimilado, transformando suas operações (Zahra \& George, 2002).

Por fim, o Gráfico 1-D mostra que o Grupo 1 apresenta respostas que estão distribuídas de 2 (raramente) a 4 (frequentemente), não havendo resposta 5 (sempre). A mediana de 3,5 indica que a parceria com a grande empresa envolve a utilização dos conhecimentos às vezes ou frequentemente. Quanto ao Grupo 2, as respostas variam de 3 (às vezes) a 5 (sempre), com mediana 4, ou seja, a utilização dos conhecimentos ocorre frequentemente. Diante disso, pode-se dizer que há uma tendência maior das startups dos programas de inovação estruturados aproveitar a parceria para utilização das informações e conhecimentos obtidos da grande empresa no desenvolvimento de novos produtos e serviços ou na melhoria do desempenho da startup, validando a hipótese 4. De fato, a competência em acessar os recursos complementares dos parceiros fornece às startups a capacidade de alavancar seus próprios recursos, o que contribui para sua vantagem competitiva e, portanto, desempenho (Wasiuzzaman, 2019).

Dada a importância das mentorias e capacitações para acelerar a aquisição de conhecimento e considerando o potencial dos processos das aceleradoras corporativas em alavancar a aprendizagem organizacional (Cajuela \& Galina, 2020), era esperado que a capacidade de absorção fosse de maior nível nas startups dos programas estruturados de inovação em comparação com aquelas dos espaços de inovação. Os resultados apresentados mostram que, de fato, os programas estruturados de inovação, por oferecerem mais sessões com mentores e treinamentos customizados para as necessidades das startups, proporcionam a elas o desenvolvimento da capacidade de absorção, tanto potencial quanto realizada. Entretanto, é importante destacar que os espaços de inovação geram aprendizagem organizacional mesmo que em menor escala, visto que também houve respostas de startups do Grupo 1 indicando que a parceria com a grande empresa trazia benefícios, sendo os mais destacados a aquisição e a assimilação de conhecimento (capacidade de absorção potencial), que apresentaram uma menor diferença entre os tipos de aceleradoras corporativas estudadas.

ISSN 2318-9231

CGE | Rio de Janeiro | v. 8 | n. 3 | set. - dez. 2020 
Esse resultado corrobora a literatura que afirma que a colaboração entre as empresas leva ao desenvolvimento de capacidade de absorção potencial, ou seja, aquisição e assimilação de conhecimentos (Fosfuri \& Tribó, 2008). Os resultados mostram que a relação entre startups e grandes empresas já resulta em capacidade de absorção potencial, especialmente a aquisição de conhecimento, mas para a capacidade de absorção realizada é preciso uma estruturação maior da parceria, que reflita a capacidade da startup em alavancar o conhecimento que foi absorvido (Zahra \& George, 2002). Isso é esperado nos programas estruturados de inovação, que promovem maior assimilação do conhecimento por incentivarem e estruturarem encontros recorrentes das startups com a grande empresa, o que resulta em uma comunicação efetiva e possibilita a internalização das informações e conhecimentos adquiridos (Cajuela \& Galina, 2020).

Da mesma forma, estudos recentes (Cajuela \& Galina, 2020; Müller, Buliga, \& Voigt, 2020) mostraram que as aceleradoras corporativas contribuem para que as startups desenvolvam principalmente capacidade de absorção potencial. Já em relação à capacidade de absorção realizada, Cajuela e Galina (2020) apontaram que as atividades de transformação e exploração do conhecimento são menos frequentes e costumam ser mais relacionadas aos interesses da corporação parceira. Tal diferença entre capacidade de absorção potencial e realizada foi observada de maneira modesta apenas para o Grupo 1, composto por startups dos espaços de inovação. Portanto, as startups dos programas estruturados de inovação têm a percepção de que o relacionamento com a grande empresa incorre em benefícios nas quatro dimensões estudadas, ou seja, tanto em termos de capacidade de absorção potencial, quanto de absorção realizada.

Quanto às desvantagens potenciais de se manter um relacionamento com uma grande empresa, foi realizado o ranqueamento das medianas e modas de cada variável, mostrado na Tabela 1, lembrando que as respostas variaram de 1 (discordo totalmente) a 5 (concordo totalmente).

Tabela 1 - Ranking da mediana e moda para cada desvantagem

\begin{tabular}{ccc}
\hline Desvantagens & Mediana & Moda \\
\hline Complex-gestão & 3,00 & 5 \\
\hline Riscos-depend & 3,00 & 3 \\
\hline Conflito-cultura & 3,00 & 2 \\
\hline Riscos-fin-org & 2,00 & 1 \\
\hline Perda-autonomia & 2,00 & 1 \\
\hline Perda-flexibilidade & 2,00 & 1 \\
\hline Implica-antitruste & 1,00 & 1 \\
\hline Perda-PI & 1,00 & 1 \\
\hline
\end{tabular}

Observou-se que as startups consideram como maior desvantagem a complexidade de gestão, a qual Barringer e Harrison (2000) descreveram como a dificuldade de gerenciar uma relação interorganizacional por exigir o esforço combinado de duas ou mais empresas e frequentemente incorrer em frustrações e atrasos onerosos. Já as outras desvantagens não foram consideradas como existentes na relação com a grande empresa, dado que as respostas foram em discordância (1 e 2) ou neutras (3). 
O fato de a complexidade de gestão ser percebida como a desvantagem mais significativa pelas startups deve-se à demora na tomada de decisão e à maior burocracia das grandes empresas, que normalmente apresentam um alto grau de departamentalização e grande quantidade de processos, em oposição à flexibilidade e agilidade das startups (Weiblen \& Chesbrough, 2015). Como indicado por esses autores, uma das motivações da aproximação das grandes empresas para com as startups é a busca por flexibilidade para conseguir rapidez para inovar. No entanto, na visão das $\operatorname{startups,~a~complexidade~de~gestão~no~relacionamento~com~a~grande~empresa~é~percebida~como~um~}$ ponto negativo.

Apesar de as startups não terem destacado as outras desvantagens estudadas, Weiblen e Chesbrough (2015) mencionam os possíveis conflitos culturais e Usman e Vanhaverbeke (2017) ressaltam que a parceria entre grandes empresas e startups, por ser assimétrica, envolve altos riscos para estas, como a perda de propriedade intelectual. Além disso, a diferença de capacidade de absorção entre a startup e a grande empresa envolvidas na parceria pode resultar em uma competição de aprendizagem entre as duas (Alvarez \& Barney, 2001), em que a corporação, por possuir vantagens de aprendizado, apresenta maior probabilidade de adotar uma postura oportunista em relação à startup (Bouncken, Gast, Kraus, \& Bogers, 2015).

Kohler (2016) ainda aponta que o relacionamento entre startups e grandes empresas, no contexto de aceleradoras corporativas, pode resultar em perda de autonomia das startups para poder pivotar e risco da startup se tornar dependente da corporação, construindo uma solução que não atende outros mercados ou não tendo a oportunidade de estabelecer parcerias com empresas que seriam concorrentes da corporação. Portanto, é importante que as startups levem em consideração essas possíveis desvantagens a fim de evitar possíveis consequências negativas para seus negócios.

\section{Considerações finais}

Este estudo teve como objetivo analisar se os diferentes tipos de apoio e serviços fornecidos pela grande empresa nas aceleradoras corporativas proporcionam às startups diferentes benefícios relacionados à capacidade de absorção. Para tanto, foram considerados dois tipos de aceleradoras, os "espaços de inovação", com serviços mais restritos e menor integração entre as organizações, e os "programas estruturados de inovação", que possibilitam maior apoio para aprofundar relacionamentos entre as organizações. Além disso, foram descritas as desvantagens decorrentes do relacionamento com uma grande empresa do ponto de vista das startups.

Os resultados mostraram que a participação das startups em qualquer dos tipos de aceleradoras corporativas gera uma aprendizagem organizacional benéfica. Entretanto, foi possível observar que os programas estruturados de inovação promovem maior capacidade de absorção para as startups, principalmente quanto à aplicação e utilização de conhecimento, que são referentes à capacidade de absorção realizada. Portanto, do ponto de vista de desenvolvimento das capacidades nas startups, é importante que sejam incentivados os programas estruturados de aceleração, que possibilitam sistematizar os serviços e processos que potencializam as relações entre as corporações e as startups. Já em relação às desvantagens, a mais destacada pelas startups foi a complexidade de gestão, o que demonstra que, na relação com uma grande empresa, destacam-se a burocracia e os atrasos na tomada de decisão.

Este estudo traz contribuições acadêmicas e práticas. Do ponto de vista acadêmico, ele contribui com o avanço do conhecimento na área específica de parcerias para inovação em ecossistemas empreendedores, uma vez que a literatura nessa área, embora crescente, ainda é insuficiente para conhecermos os benefícios dos ambientes de apoio para as startups. Dessa forma, este trabalho agrega à literatura mais informações sobre a perspectiva das startups quanto às parcerias estabelecidas com grandes empresas em aceleradoras corporativas, e, em específico, também permite avançar os estudos sobre capacidade de absorção em alianças interorganizacionais, ainda apontados como incipientes (Fosfuri \& Tribó, 2008; Cajuela \& Galina, 2020). Do ponto de vista das desvantagens da parceria, podemos dizer que este trabalho é um dos pioneiros no Brasil ao mostrar uma avaliação do ponto de vista das startups, apresentando assim uma lente de análise ainda para ser explorada.

A principal contribuição prática deste estudo é mostrar claramente que programas estruturados trazem mais benefícios para o desenvolvimento das startups, o que permite orientar práticas dos ambientes de estímulo a 
empresas nascentes, em específico das aceleradoras corporativas. Ademais, os resultados desta pesquisa são de interesse para as grandes corporações que pretendem estruturar ou participar de aceleradoras corporativas, focando nos serviços e tipos de apoio que podem ser oferecidos às startups de maneira a construir uma relação interorganizacional que seja benéfica para ambas as partes; e para as startups que pretendem analisar a entrada em diferentes aceleradoras corporativas, sendo possível compreender os benefícios e desvantagens envolvidos nesse tipo de relação interorganizacional.

Este estudo teve limitações, que merecem ser citadas. Uma delas é o teor descritivo das análises feitas, visto que os dados obtidos não nos permitiram ampliar a avaliação com utilização de técnicas estatísticas mais complexas. Assim, é necessário ampliar a amostra com dados mais diversos tanto de aceleradoras quando de startups, o que possivelmente permitirá uma variedade maior de relacionamentos entre elas e, consequentemente, indicadores pertinentes para usar estatística mais complexa. Outra limitação deste estudo é a realização de uma coleta única de dados, seria importante acompanhar essas empresas tanto para avaliar os benefícios quanto as dificuldades do ponto de vista das startups. Quanto ao aspecto exploratório deste estudo sobre as desvantagens das parcerias apontadas pelas startups, os resultados podem indicar uma via de análises futuras em estudos organizacionais. É importante destacar que o fato das startups não identificarem certas desvantagens que são mencionadas na literatura possivelmente está relacionado à limitação do estudo de fazer um recorte no tempo e não acompanhar as várias fases de desenvolvimento das empresas. Diante disso, estudos futuros poderiam analisar se a percepção das startups quanto às desvantagens decorrentes do envolvimento com grandes empresas varia de acordo com o tempo: uma hipótese seria que as desvantagens podem se tornar mais evidentes conforme a duração do relacionamento com uma grande empresa aumenta. Além disso, há espaço para analisar os benefícios e desvantagens dentro de uma perspectiva de longo prazo (Crișan et al., 2019), bem como compreender o quanto estes fatores influenciam a escolha das startups em participar de uma aceleradora corporativa.

\section{Referências}

Abreu, P. R. M., \& Campos, N. M. (2016). O panorama das aceleradoras de startups no Brasil. North Charleston, EUA: CreateSpace Independent Publishing Platform. https://bibliotecadigital.fgv.br/dspace/bitstream/handle/10438/18853/Abreu\%3B\%20Campos\%20Neto_Pano rama $\% 20 \mathrm{das} \% 20$ aceleradoras $\% 20 \mathrm{de} \% 20$ startups $^{2} \% 20$ no $^{2} \% 20$ Brasil.pdf? sequence $=1$ \&is Allowed $=\mathrm{y}$

Alvarez, S., \& Barney, J. B. (2001). How entrepreneurial firms can benefit from alliances with large partners. The Academy of Management Executive, 15(1), 139-148.

Barringer, B. R., \& Harrison, J. S. (2000). Walking a tightrope: Creating value through interorganizational relationships. Journal of Management, 26(3), 367-403.

Becker, W., \& Dietz, J. (2004). R\&D cooperation and innovation activities of firms - Evidence for the German manufacturing industry. Research Policy, 33(2), 209-223.

Boone, H. N., \& Boone, D. A. (2012). Analyzing Likert Data. Journal of Extension, 50(2).

Bouncken, R. B., Gast, J., Kraus S., \& Bogers, M. (2015). Coopetition: A systematic review, synthesis, and future research directions. Review of Managerial Science, 9(3), 577-601.

Brink, T. (2017). SME routes for innovation collaboration with larger enterprises. Industrial Marketing Management, $64,122-134$.

Cajuela, A. R., \& Galina, S. V. R. (2020). Processos em relacionamentos interorganizacionais para desenvolvimento de capacidade de absorção em startups. Revista de Administração Contemporânea, 24(6), 550-566.

Choi, Y., \& Kim, D. (2018). The effects of investor types on investees' performance: Focusing on the seed accelerator. Cogent Economics \& Finance. https://doi.org/10.1080/23322 039.2018.15508 70 
Cohen, S., Fehder, D. C., Hochberg, Y. V., \& Murray, F. (2019). The design of startup accelerators. Research Policy, 48(7), 1781-1797. https://doi.org/10.1016/j.respo 1.2019.04.003

Cohen, S., \& Hochberg, Y. V. (2004, March 30). Accelerating startups: The seed accelerator phenomenon. 2-10. Available at SSRN: https://ssrn.com/abstract $=2418000$

Cohen, W. M., \& Levinthal, D. A. (1990). Absorptive capacity: A new perspective on learning and innovation. Administrative Science Quarterly, 128-152.

Crișan, E. L. et al. (2019). A systematic literature review on accelerators. The Journal of Technology Transfer, 1-28.

Davis, J. P. (2016). The group dynamics of interorganizational relationships: Collaborating with multiple partners in innovation ecosystems. Administrative Science Quarterly, 61(4), 621-661.

Dooley, L., Kenny, B., \& Cronin, M. (2015). Interorganizational innovation across geographic and cognitive boundaries: Does firm size matter?. R\&D Management, 46(S1), 227-243.

Evangelista, F., \& Mac, L. (2016). The influence of experience and deliberate learning on SME export performance. International Journal of Entrepreneurial Behavior \& Research, 22(6), 860-879. https://doi.org/10.1108/IJEBR-12-2015$\underline{0300}$

Fosfuri, A., \& Tribó, J. A. (2008). Exploring the antecedents of potential absorptive capacity and its impact on innovation performance. Omega, 36, 173-187.

Groote, J. K., \& Backmann, J. (2020). Initiating open innovation collaborations between incumbents and startups: How can David and Goliath get along?. International Journal of Innovation Management, 24(2), 2050011. https://doi.org/10.1142/S1363919620500115

Jang, H., Lee, K., \& Yoon, B. (2017). Development of an open innovation model for R\&D collaboration between large firms and small-medium enterprises (SMES) in manufacturing industries. International Journal of Innovation Management, 21(1), 1750002.

Kohler, T. (2016). Corporate accelerators: Building bridges between corporations and startups. Business Horizons, 59(3), 347-357.

Liu, H.-M., \& Yang, H.-F. (2019). Managing network resource and organizational capabilities to create competitive advantage for SMEs in a volatile environment. Journal of Small Business Management, 57(S2), 155-171, https://10.1111/jsbm.12449

Moschner, S. et al. (2019). Toward a better understanding of corporate accelerator models. Business Horizons, 62(5), 637-647.

Müller, J. M., Buliga, O., \& Voigt, K. I. (2020, Feb. 1st). The role of absorptive capacity and innovation strategy in the design of industry 4.0 business models - A comparison between SMEs and large enterprises. European Management Journal. https://doi.org/10.1016/j.emj.2020.01.002

Pauwels, C. et al. (2016). Understanding a new generation incubation model: The accelerator. Technovation, 50-51, 13-24.

Pavlou, P. A., \& El Sawy, O. A. (2006). From IT leveraging competence to competitive advantage in turbulent environments: The case of new product development. Information Systems Research, 17(3), 198-227, 2006.

Shankar, R. K., \& Shepherd, D. A. (2019). Accelerating strategic fit or venture emergence: Different paths adopted by corporate accelerators. Journal of Business Venturing, 34(5), 105886. https://doi.org/10.1016/j.jbusvent.2018.06.004

Simon, H., \& Leker, J. (2016). Using startup communication for opportunity recognition: An approach to identify future product trends. International Journal of Innovation Management, 20(8), 1640016-1-1640016-22. 
Sommer, L. P., Heidenreich, S., \& Handrich, M. (2017). War for talents: How perceived organizational innovativeness affects employer attractiveness. R\&D Management, 47(2), 299-310.

Stuart, T. E. (2000). Interorganizational alliances and the performance of firms: A study of growth and innovation rates in a high-technology industry. Strategic Management Journal, 21(8), 791-811.

Urbaniec, M.; Żur, A. (2020). Business model innovation in corporate entrepreneurship: Exploratory insights from corporate accelerators. International Entrepreneurship and Management Journal, 1-24.

Usman, M., \& Vanhaverbeke, W. (2017). How start-ups successfully organize and manage open innovation with large companies. European Journal of Innovation Management, 20(1), 171-186.

Wasiuzzaman, S. (2019). Resource sharing in interfirm alliances between SMEs and large firms and SME access to finance. Management Research Review, 42(12), 1375-1399.

Weiblen, T., \& Chesbrough, H. W. (2015). Engaging with startups to enhance corporate innovation. California Management Review, 57(2), 66-90.

Zahra, S. A., \& George, G. (2002). Absorptive capacity: A review, reconceptualization, and extension. Academy of Management Review, 27(2), 185-203. https://doi.org/10.5465/AMR.2002.6587995 